\title{
Laser-Induced Fluorescence Determination of Flame Temperatures in Comparison with CARS Measurements
}

\author{
A. Lawitzki, I. Plath, W. Stricker, J. Bittner, U. Meier, and K. Kohse-Höinghaus \\ DLR-Institut für Physikalische Chemie der Verbrennung, Pfaffenwaldring 38-40, \\ D-7000 Stuttgart 80, Fed. Rep. Germany
}

Received 27 December 1989/Accepted 20 March 1990

\begin{abstract}
Temperature profiles in several premixed low pressure $\mathrm{H}_{2} / \mathrm{O}_{2} / \mathrm{N}_{2}$ flames and in an atmospheric pressure $\mathrm{CH}_{4}$ /air flame were determined by laser-induced fluorescence (LIF) and by CARS experiments. In the LIF study, temperatures were derived from $\mathrm{OH}$ excitation spectra, CARS temperatures were deduced from $\mathrm{N}_{2} Q$-branch spectra. The present study is the first quantitative comparison of these two methods for temperature determination in flames burning at pressures up to 1 bar. The resulting temperatures showed good agreement.
\end{abstract}

PACS: $82.40 \mathrm{Py}, 33.50 \mathrm{Dq}$

Detailed chemical kinetic modelling requires reliable experimental data for comparison with the model predictions. The knowledge of the species concentrations and the gas temperature in a flame contributes to the understanding of the chemistry of combustion processes. Temperature is one of the most important quantities in flame studies: it is needed to determine the total mole fraction of the reactive radicals under investigation, and the temperatures at different locations in the flame are necessary as input parameters for the model calculation. Many investigations used different optical methods to measure temperatures in flames. The advantage of these techniques over intrusive probe measurements is the fact that they can be applied without disturbing the combustion process. Eckbreth [1] and Laurendeau [2] give a good review of the recent progress in this field.

In conjunction with measurements of radical concentrations by laser-induced fluorescence (LIF), it is useful to employ the same technique (rather than CARS or other processes) for the complementary temperature measurements. Basically, there are two approaches to the problem. Fluorescence spectra may be used to determine excited electronic state populations, but they require excessive modelling of the different collision processes involved [3-5]. Excitation spectra yield ground state population distributions which more likely reflect the true gas temperature.

Recently, strategies for reliable LIF temperature determination have been proposed [6]. In this investigation, two LIF methods were used which differ mainly in the laser power density used for the excitation. In the "linear excitation method", the fluorescence intensity depended linearly on the laser power density. The "nonlinear excitation method" [7] made use of partially saturated laser-induced fluorescence: the laser power density was increased to a regime where the fluorescence intensity was no longer linearly dependent on the laser power density. Temperature measurements with these two excitation methods have been compared in a variety of systems with different temperature and chemical environment; in general, good agreement was found [6]. An additional validation of the LIF results by independent optical techniques as CARS or Raman methods is attempted in this study.

Up to now, there have been only few investigations of LIF temperature measurements in comparison with other independent measuring techniques. Some years ago, Bechtel [8] presented temperature profiles in an 
atmospheric pressure $\mathrm{CH}_{4}$ /air flame measured by laser-induced fluorescence and by laser Raman scattering. In a very recent investigation, LIF measurements of $\mathrm{OH}$ in $\mathrm{CH}_{4}$ /air flames at higher pressures up to 10 bar were compared with resonance CARS experiments [9].

LIF is often chosen as a diagnostic tool for combustion processes because of its two-dimensional mapping capabilities. But the LIF technique may be handicapped by the influence of gas kinetic collisions, especially at higher pressures. In contrast to that, the CARS technique is not influenced by quenching. CARS is a well-established technique for the determination of temperatures, particularly in hostile, technical combustion environments. Like the laser-induced fluorescence, the CARS process enables spatially precise time-resolved measurements in a single shot technique. With both methods, temperatures can be derived from rotational population distributions.

In this study, laser-induced fluorescence has been used to determine temperatures from rotational excitation spectra probing the electronic ground state of $\mathrm{OH}$ radicals, whereas CARS temperatures were derived from $\mathrm{N}_{2} Q$-branch spectra. The experiments were performed in several premixed low pressure $\mathrm{H}_{2} / \mathrm{O}_{2} / \mathrm{N}_{2}$ flames and in a $\mathrm{CH}_{4} /$ air flame at atmospheric pressure. This study presents the first quantitative comparison of temperature measurements in low pressure flames with the two independent diagnostic techniques LIF and CARS.

\section{Experimental}

The experimental setup, as far as the LIF measurements are concerned, has been described earlier [6]. The main features shall be repeated briefly.

$\mathrm{OH}$ radicals were excited in the $\left(A^{2} \Sigma^{+}-X^{2} \Pi, 1-0\right)$ band with a frequency-doubled, Nd:YAG-pumped dye laser (Spectra Physics) using pulse energies of $\approx 1 \mathrm{~mJ}$ at $280 \mathrm{~nm}$ and a spectral bandwidth of $\approx 0.7 \mathrm{~cm}^{-1}$. All temperatures were determined from excitation spectra, where the laser was scanned across different $\mathrm{OH}$ rotational transitions in the $S$-branch or in the $P$-and $R$-branches. For the nonlinear excitation method, the laser was focussed by a $f=200 \mathrm{~mm}$ lens, providing a spatial resolution of $100-200 \mu \mathrm{m}$. In the case of linear excitation, the unfocussed laser had to be attenuated by a factor of nearly 100 to ensure that the fluorescence signal depended linearly on the laser power density. Spatial resolution was provided by a $0.65 \mathrm{~mm}$ diameter pinhole in the laser beam. The laser intensity was monitored with a calibrated vacuum photodiode in both cases.

For both types of excitation, the fluorescence was collected with a $f=100 \mathrm{~mm}$ lens at right angle to the laser beam direction. Spectral resolution of the fluorescence was provided by a broadband interference filter ( $35 \mathrm{~nm}$ FWHM, centered at $318 \mathrm{~nm}$ ). The bandpass of the filter covered the entire (1-1) and (0-0) bands. The fluorescence intensity was detected with a fast photomultiplier and processed with a gated integrator (Stanford Research System) using different sampling gates between 5 and $30 \mathrm{~ns}$. Typically, 10-30 pulses were averaged for one spectral position.

For the determination of temperatures from CARS spectra, the following experimental setup was used. A multimode frequency-doubled $\mathrm{Nd}: \mathrm{YAG}$ laser (Lumonics) delivered up to $280 \mathrm{~mJ} /$ pulse at $532 \mathrm{~nm}$. Approximately $65 \%$ of the power was used to pump a broadband dye laser (FWHM: $148 \mathrm{~cm}^{-1}$ ) centered at $607 \mathrm{~nm}$, which served as the Stokes beam for the CARS process. The residual power at $532 \mathrm{~nm}$ was used as the pump frequency. The pump and Stokes beams were focussed in a planar BOXCARS geometry [10] with a lens of $300 \mathrm{~mm}$ focal length, forming a crossing half angle of about $2.4^{\circ}$. The spatial resolution was about $1.5 \mathrm{~mm}$ in length for the generation of $90 \%$ of the CARS signal. The detection system included a double monochromator (SPEX Model 1403), an intensified photodiode array camera (PARC 1421) and a detector controller (PARC 1460). The dispersion of the system was determined using the line spectrum of a Cr hollow cathode lamp between 460 and $475 \mathrm{~nm}$. The exact peak positions were calculated assuming a Lorentzian line shape. The result was an overall dispersion of $0.30 \mathrm{~cm}^{-1} / \mathrm{pixel}$. The dispersion varied over the $25 \mathrm{~mm}$ length of the diode array. Therefore the mean dispersion in the region of the CARS spectrum was evaluated by fitting a flame spectrum yielding a dispersion of $0.298 \mathrm{~cm}^{-1} /$ pixel with an accuracy of $0.3 \%$.

For the CARS data generation in flames at atmospheric pressure, 600 pulses were averaged, whereas at 95 mbar, 200-1600 pulses were averaged depending on the temperature. To correct the spectra for the spectral intensity distribution of the dye laser, for the transmission characteristics of all optical elements, and for the detector sensitivity, the spectra were divided by a normalized non-resonant CARS spectrum generated in $\mathrm{CO}_{2}$. The laser energies at $532 \mathrm{~nm}$ were varied between 1 and $60 \mathrm{~mJ}$ to match the dynamic range of the diode array.

In both types of experiments, LIF as well as CARS, the same low pressure burner and the same atmospheric pressure burner were used. The low pressure $\mathrm{H}_{2} / \mathrm{O}_{2} / \mathrm{N}_{2}$ flames were supported on a flat flame burner with a brass plate of $4.2 \mathrm{~cm}$ diameter in an evacuated housing. Details concerning this burner can be found elsewhere [11]. The flame conditions were the same as for the $\mathrm{H}_{2} / \mathrm{O}_{2} / \mathrm{Ar}$ flames at $95 \mathrm{mbar}$ described earlier [12], with the dilution gas argon replaced by 
nitrogen. The flow velocity of the cold gases was $31 \mathrm{~cm} / \mathrm{s}$. The $\mathrm{H}_{2} / \mathrm{O}_{2} / \mathrm{N}_{2}$ flames were run at three different stoichiometries: $\phi=0.6,1.0$, and 1.4.

For the investigation of a stoichiometric $\mathrm{CH}_{4} /$ air flame at 1 bar, a McKenna burner with a $6 \mathrm{~cm}$ diameter porous plug was used. The burner could be moved vertically and horizontally. For most measurements, the laser beam traversed the burner at the center. The premixed cold gases had a flow velocity of $19.2 \mathrm{~cm} / \mathrm{s}$.

\section{Results and Discussion}

Temperatures in $\mathrm{N}_{2}$-containing flames at $95 \mathrm{mbar}$ and 1 bar have been determined by both LIF and CARS. In the following, some important aspects of these LIF and CARS experiments will be discussed, and, finally, the resulting temperatures will be compared.

\subsection{Temperature Determination by $L I F$}

Suitable strategies for the determination of the temperatures under a variety of conditions by laser-induced fluorescence have been described previously $[6,9]$. Some essentials concerning the present LIF measurements are briefly addressed.

Problems due to rotational-level-dependent fluorescence quantum yields, absorption of laser light by the flame gases and fluorescence trapping can influence the temperature measurements. To minimize errors caused by these effects, an appropriate spectral region for excitation and detection transitions has to be chosen. Here, $\mathrm{OH}$ radicals were excited in the (1-0) band of the $A-X$ system. The fluorescence was collected with a broad detection bandpass in the (1-1) and $(0-0)$ bands. Different effective lifetimes and thus different fluorescence quantum yields for different excited transitions make it preferable to use a detection time interval which is short compared to the fluorescence lifetime of the radical and which begins early after the laser pulse. Then only few collisions of the excited $\mathrm{OH}$ radicals have taken place. This approach was possible only for the low pressure flames. In the atmospheric pressure flame, where the fluorescence lifetime was shorter than the laser pulse, only temporally integrated fluorescence intensities could be measured.

For the excitation of the $\mathrm{OH}$ radicals, suitable transitions were chosen. The upper half of Fig. 1 shows part of an $\mathrm{OH}$ excitation spectrum. A combination of the $R_{2}(13), P_{1}(2)$, and $R_{1}(14)$ lines near $282.6 \mathrm{~nm}$ allows a comparatively rapid measurement of an excitation spectrum. All three lines are single lines; they cover a large energy difference between ground state rota-

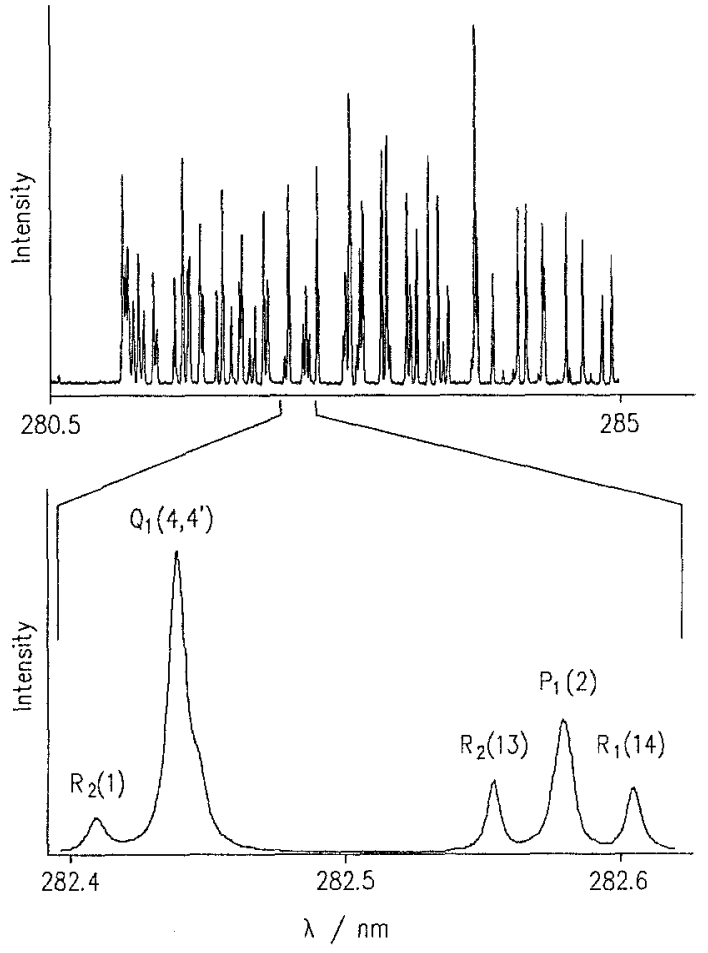

Fig. 1. $O H$ excitation spectra in the $(1-0)$ band of the $A-X$ system. In the lower part several $P, Q, R$ lines are shown in detail

tional levels within a short wavelength range. This range is shown in the lower part of Fig. 1. For temperatures above $1000 \mathrm{~K}$, these three lines offer a good temperature sensitivity. In the atmospheric pressure flame, some absorption of the laser light $(<10 \%)$ was noticed for the $P_{1}(2)$ line. This had to be taken into account for the evaluation of temperatures from the measured fluorescence intensities.

The influence of laser absorption on the temperature measurement was substantially reduced by excitation of $\mathrm{OH}$ in the (1-0) $S_{21}$-branch, which has a comparatively low transition probability. The $S$-branch appeared well suited because of its widely separated, isolated lines [6]. In the atmospheric pressure flame, temperatures were mostly obtained from $S$-branch spectra, whereas in the low pressure flames, temperatures were obtained from both $S$-branch spectra and the $P$ and $R$ transitions mentioned above.

Two methods of excitation have been used in the LIF experiments. In the linear excitation method, the linear dependence of the fluorescence intensity on the laser power density had to be controlled carefully. This method is rather simple, especially in the evaluation of the data from the recorded spectra. But the spatial resolution is limited, since it is provided by a pinhole in the unfocussed laser beam.

Nevertheless, a high spatial resolution is necessary for an accurate temperature determination particular- 
ly in the flame front. Therefore the method of nonlinear excitation $[6,7]$ was applied in addition. In this case, a much higher laser power density was used for the excitation. This has the advantage of generating high fluorescence intensities which permit a higher spatial resolution and enhance signal-to-noise ratios. However, the method based on nonlinear excitation is slightly more complicated, as the degree of saturation has to be considered in addition. In order to measure reliable temperatures, a moderate degree of saturation corresponding to an appropriate region of the laser power density had to be chosen [13]. Up to now, all measurements with the nonlinear approach were performed in the $S$-branch.

Temperatures were derived from Boltzmann plots, where the normalized fluorescence intensity divided by the line strength and degeneracy was plotted versus rotational energy of the electronic ground state. In all cases good least squares fits resulted with a statistical error of less than $2 \%$ for a single temperature measurement.

\subsection{Temperature Determination by $C A R S$}

The CARS spectra were analyzed using the computer code CARP-3 [14] to deduce the $\mathrm{N}_{2}$ temperatures. The code accounts for both the motional narrowing and cross coherence effects. At low pressures below 1 bar, the code treats the combined effect of pressure and Doppler broadening of the linewidths assuming a Voigt profile. Only the temperature and the spectral shift of the spectrum were fitted. The nitrogen concentration and also the non-resonant susceptibility were kept fixed during the fit. The instrument function was obtained daily by fitting carefully measured room temperature CARS spectra.

Recently, a nonlinear response of intensified photodiode array detectors at low intensities has been detected $[15,16,17]$. This nonlinearity had to be taken into account to avoid a systematic error in the CARS temperatures. The linearity characteristics of the detection system used here was studied with the laser light at $532 \mathrm{~nm}$ which illuminated heights of 1.4 and $2.5 \mathrm{~mm}$ of the diode array. The results were in agreement with the observations of Snelling et al. [16] and Kröll et al. [17] showing a reduced gain at lower number of counts. No saturation of the diodes was observed up to 12000 counts. A logarithmic function was fitted to measured data points and used to calculate a correction factor for the observed intensities in the resonant and nonresonant CARS spectra. The temperatures of the corrected spectra were at 1 bar and $2000 \mathrm{~K}$ about $50 \mathrm{~K}$ and at 95 mbar and $1400 \mathrm{~K}$ about $38 \mathrm{~K}$ higher than for the uncorrected case.
The population of higher vibrational levels by stimulated Raman scattering might cause a systematic error in the CARS temperatures $[18,19]$. In some of the low pressure CARS spectra taken with $60 \mathrm{~mJ} /$ pulse in the pump beams, a small systematic deviation in the $v=1-2$ band could not be fitted keeping the rotational and vibrational temperature equal. To avoid any influence of saturation only the $v=0-1$ band was fitted. The evaluated temperatures were lowered by about $7 \mathrm{~K}$.

\subsection{Comparison of $L I F$ and CARS Temperatures}

In the following, a comparison of the temperatures determined under the same flame conditions with LIF and CARS is given. In the low pressure $\mathrm{H}_{2} / \mathrm{O}_{2} / \mathrm{N}_{2}$ flames, temperatures were measured at different heights above the burner surface. Temperature profiles in the rich, the stoichiometric, and the lean $\mathrm{H}_{2} / \mathrm{O}_{2} / \mathrm{N}_{2}$ flame are given in Fig. 2. Both the results from linear and nonlinear excitation spectra agree well. The agreement with CARS temperatures is best in the rich flame. In the $\phi=0.6$ and the $\phi=1.0$ flames, the CARS temperatures appear systematically higher than the LIF temperatures. A large number of temperatures was measured in the lean $\mathrm{H}_{2} / \mathrm{O}_{2} / \mathrm{N}_{2}$ flame at a height of $15 \mathrm{~mm}$ above the burner surface. A mean temperature of $(1360 \pm 30) \mathrm{K}$ resulted with the LIF method and $(1415 \pm 25) \mathrm{K}$ were obtained from CARS measurements; the errors are the deviations from the mean values $(1 \sigma)$. Temperature profiles like those shown in Fig. 2 may be used as input for a detailed flame modelling.

The temperature measurements in the $\mathrm{H}_{2} / \mathrm{O}_{2} / \mathrm{N}_{2}$ flames can be compared with temperatures obtained recently with $\mathrm{LIF}$ in $\mathrm{H}_{2} / \mathrm{O}_{2} / \mathrm{Ar}$ flames at $95 \mathrm{mbar}$ [6]. The temperatures in the lean and stoichiometric $\mathrm{H}_{2} / \mathrm{O}_{2} / \mathrm{N}_{2}$ flames are about $100 \mathrm{~K}$ lower than in the corresponding $\mathrm{H}_{2} / \mathrm{O}_{2} / \mathrm{Ar}$ flames; in the rich flames, the temperatures differ by about $50 \mathrm{~K}$.

The stoichiometric $\mathrm{CH}_{4} /$ air flame at atmospheric pressure was investigated with both techniques, LIF and CARS. Here, a further independent cross check was obtained from Raman measurements. Table 1 compares the temperatures determined in the present study with literature data from similar flames. The temperatures from LIF, CARS and Raman spectra were measured at a height of $4 \mathrm{~mm}$ above the burner surface. In this region, the temperature does not vary with the distance from the burner surface. The temperature profile in the 1 bar $\mathrm{CH}_{4}$ /air flame is flat above $2 \mathrm{~mm}$, as determined from CARS spectra. All temperatures listed in Table 1 show very good agreement. From LIF linear excitation spectra, a mean tempera- 


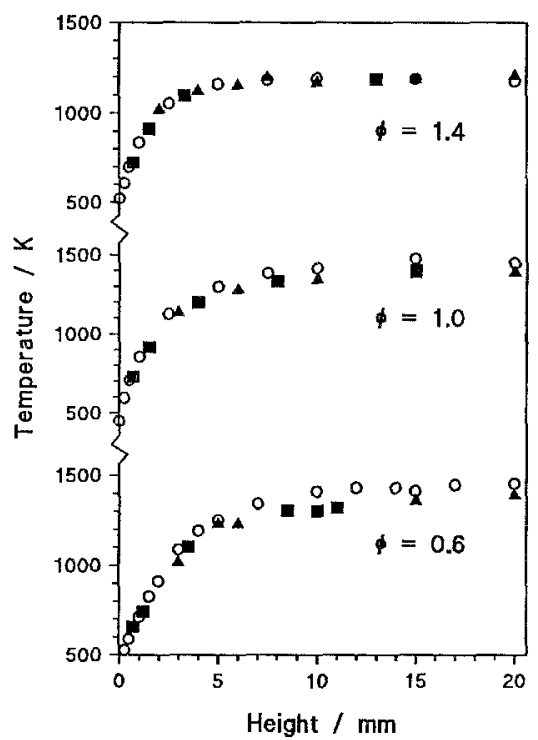

Fig. 2. Temperature profiles versus height above the burner surface in $\mathrm{H}_{2} / \mathrm{O}_{2} / \mathrm{N}_{2}$ flames at $95 \mathrm{mbar}$ for three different stoichiometries. The filled triangles represent temperatures from linear excitation, the filled squares result from nonlinear excitation, and the open circles represent temperatures from CARS spectra. Typical errors are smaller than the size of the symbols

Table 1. Comparison of temperatures in the postflame region of stoichiometric $\mathrm{CH}_{4}$ /air flames at 1 bar measured with different techniques. The first three temperatures were measured using the same burner under exactly the same flame conditions. The last six values are temperatures from the literature, which have been determined in stoichiometric $\mathrm{CH}_{4}$ /air flames at very similar cold gas velocities

\begin{tabular}{ll}
\hline Method & Temperature [K] \\
\hline LIF (this work) & $2100 \pm 70$ \\
CARS (this work) & $2065 \pm 50$ \\
Raman (this work) & $2030 \pm 40$ \\
Absorption spectra [20] & $2000 \pm 100$ \\
Degenerate four-wave mixing [21] & $2100 \pm 110$ \\
Resonance CARS [22] & $2200 \pm 100$ \\
LIF [9] & $2040 \pm 50$ \\
CARS [23] & $2000 \pm 50$ \\
Raman [23] & $2070 \pm 60$ \\
\hline
\end{tabular}

ture of $(2100 \pm 70) \mathrm{K}$ resulted from a large number of measurements. This agrees very well with the CARS and Raman temperatures in the same flame. For a further comparison, temperatures measured in very similar flames are shown in Table 1. Here, techniques as laser absorption and degenerate four-wave mixing were used in stoichiometric $\mathrm{CH}_{4}$ /air flames with similar cold gas flow velocities as in the present study. A comparison between resonance CARS and linear excitation LIF temperatures in $\mathrm{CH}_{4}$ /air flames at higher pressures up to 10 bar yielded equally good agreement [9]. An interesting aspect for further studies is the extension of the pressure range. CARS and Raman measurements have already been performed in laminar, flat $\mathrm{CH}_{4}$ /air flames at pressures up to $40 \mathrm{bar}$ [23]. Complementary temperature measurements with LIF under the same flame conditions are projected. The present investigation, as well as the recent study with measurements up to 10 bar [9] can serve as a basis for such experiments.

\section{Summary}

Strategies for the accurate determination of temperatures with laser-induced fluorescence have been presented. For reasons of comparisons CARS measurements were performed. Several premixed low pressure $\mathrm{H}_{2} / \mathrm{O}_{2} / \mathrm{N}_{2}$ flames were investigated, and the first quantitative comparison of these two different diagnostic techniques in this pressure range yielded good agreement. In the 1 bar $\mathrm{CH}_{4}$ /air flame, LIF and CARS temperatures were compared with independent temperature measurements from Raman spectra. All three temperatures agree very well; in addition, they are in good agreement with literature data.

Acknowledgements. The authors thank Prof. Dr. Th. Just for his continuous interest and support of this study. This work was financially supported by the Bundesministerium für Forschung und Technologie, and the Arbeitsgemeinschaft TECFLAM.

\section{References}

1. A.C. Eckbreth: Laser Diagnostics for Combustion Temperature and Species (Abacus, Tunbridge Wells 1988)

2. N.M. Laurendau: Prog. Energy Combust. Sci. 14, 147 (1988)

3. C. Chan, J.W. Daily: Appl, Opt. 19, 1963 (1980)

4. Y. Furuya, M. Yamamoto, Y. Takubo: Jpn. J. Appl. 24, 455 (1985)

5. G. Zizak, J.A. Lanauze, J.D. Winefordner: Combust. Flame 65, 203 (1986)

6. A. Lawitzki, R. Tirgrath, U. Meier, K. Kohse-Höinghaus, A. Jörg, Th. Just: Proceedings of the Joint Meeting of the German and Italian Sections of the Combustion Institute, Ravello, Italy 1989, p. 1.4

7. R. Tirgrath: Thesis, DLR Stuttgart (in preparation)

8. J.H. Bechtel: Appl. Opt. 18, 2100 (1979)

9. K. Kohse-Höinghaus, U. Meier, B. Attal-Trétout: Appl. Opt. 29 (1990), to be published

10. A.C. Eckbreth: Appl. Phys. Lett. 32, 421 (1978)

11. K. Kohse-Höinghaus, P. Koczar, Th. Just: Twenty-First Symposium (International) on Combustion (The Combustion Institute 1986) p. 1719

12. K. Kohse-Höinghaus, S. Kelm, U. Meier, J. Bittner, Th. Just: In Complex Chemical Reaction Systems ed. by J. Warnatz, W. Jäger (Springer, Berlin, Heidelberg 1987) pp. 292-301 
13. A. Lawitzki, R. Tirgrath, U. Meier, K. Kohse-Höinghaus: (in preparation)

14. CARP-3 is under the copyright of UKEA Harwell, England

15. D.R. Snelling, G.J. Smallwood, T. Parameswaran: Appl. Opt. 28, 3233 (1989)

16. D.R. Snelling, G.J. Smallwood, R.A. Sawchuk: Appl. Opt. 28, 3226 (1989)

17. S. Kröll, M. Aldén, P.-E. Bengtsson, C. Löfström: Appl. Phys. B 49, 445 (1989)

18. M. Péalat, M. Lefebvre, J.P. Taran, P.L. Kelley: Phys. Rev. A 38, 1948 (1988)
19. A. Gierulski, M. Noda, T. Yamamoto, G. Marowsky, A. Slenczka: Opt. Lett. 12, 608 (1987)

20. R.J. Cattolica: Combust. Flame 44, 43 (1982)

21. T. Dreier, CRF, Sandia National Laboratory, Livermore, USA: Private communication, 1989

22. B. Attal-Trétout, S.C. Schmidt, E. Crété, P. Dumas, J.P. Taran: J. Quant. Spectrosc. Radiat. Transfer, to be published (1990)

23. W. Stricker, M. Woyde: Proceedings of the Joint Meeting of the German and Italian Sections of the Combustion Institute, Ravello, Italy 1989 , p. 1.1 